\title{
Numerical investigation of plasmonic photonic hybrid whispering gallery modes
}

\author{
Aneesh V. Veluthandath ${ }^{1 *}$, Shubhayan Bhattacharya ${ }^{1}$, Ganapathy Senthil Murugan ${ }^{2}$, James S. \\ Wilkinson $^{2}$, Prem B. Bisht ${ }^{1}$ \\ ${ }^{1}$ Department of Physics, Indian Institute of Technology, Madras, Chennai, India -600036 \\ ${ }^{2}$ Optoelectronics Research Centre, University of Southampton, Southampton, UK-SO17 1BJ \\ *aneesh@physics.iitm.ac.in
}

\begin{abstract}
The hybrid plasmonic photonic WGM modes of a metal coated microsphere resonator are numerically investigated. The proposed geometry consists of a three-layer structure with a silica microsphere core, a $30 \mathrm{~nm}$ silver intermediate layer and an outer polymer layer. It has been shown that the proposed geometry supports hybrid resonances with moderately high $Q$-factor and low mode volume. The properties of the hybrid modes can be tuned by varying the thickness of the polymer layer.
\end{abstract} modes

Keywords- Plasmonics: microcavity: whispering galley

\section{INTRODUCTION}

Enhancing the light matter interaction is required to achieve efficient nanophotonic devices such as single photon sources and low threshold microlasers. In resonators one of the most important parameter that characterizes the light matter interaction is the Purcell factor which is proportional to ratio of the quality factor $(\mathrm{Q})$ of the resonance and the mode volume $\left(\mathrm{V}_{\text {eff }}\right)$ - the volume occupied by the optical mode of the cavity. Higher $\left(\mathrm{Q} / \mathrm{V}_{\text {eff }}\right)$ can be can be achieved by using a cavity with very high $\mathrm{Q}$ such as whispering gallery modes (WGM) resonance of a microsphere or a cavity with very low mode volume such as plasmonic cavities. The mismatch of the WGM resonance frequency and the natural linewidth of the quantum emitter limits the maximum achievable Purcell factor of WGM resonators below 100 [1]. In contrast, plasmonic microcavities with Purcell factor as high as 2000 have been reported [1]. However, due to the low Q values of the plasmonic resonances, it is not possible to use plasmonic cavities for single frequency devices. Therefore, it was suggested that, a high performance device with narrow bandwidth can be achieved by combining the properties of plasmonic and WGM cavities.

Plasmonic WGM resonators such a metal-coated disc resonators [2] and microbottle resonators [3] have a metallic outer layer which makes the coupling of the light in and out of the cavity difficult. Therefore, in this study we propose a three layer plasmonic photonic WGM resonator with a silica core, an intermediate plasmonic layer and an outer polymethyl methacrylate (PMMA) layer. The hybrid mode that is localized in the outer dielectric layer can be easily coupled into and out of the cavity using a tapered fiber coupler. Furthermore, PMMA is a good host for active emitters such as semiconductor quantum dots and organic dyes, so that active devices can be fabricated by doping the PMMA layer with these emitters.

\section{NUMERICAL MODELING OF HYBRID WGM RESONATORS}

\section{A. Computational method}

The axisym method [4] implemented in Comsol Multiphysics was used to estimate the resonance frequency and mode field distributions of the hybrid modes. The Qfactor of the cavity was estimated from the complex eigenvalue $(\omega)$ of the resonance with

$$
Q=\frac{\mathfrak{R} e(\omega)}{2 \mathfrak{I m}(\omega)}
$$

The $\mathrm{V}_{\text {eff }}$ of the particular mode in consideration, was estimated using eq. 2

$$
V_{e f f}=\frac{\int_{V} \varepsilon(r)|E(r)|^{2} d V}{\max \left[\varepsilon(r)|E(r)|^{2}\right]}
$$

Where $\varepsilon(r)$ is the dielectric constant, $|E(r)|$ is the electric field strength and $V$ is the quantization volume of the resonator.

\section{B. Hybrid WGM modes}

The proposed cavity consists of a silica microsphere of radius $30 \mu \mathrm{m}$ on which a $30 \mathrm{~nm}$ silver layer is coated. A third layer, of PMMA, was added on top of the silver nanolayer. The refractive index of the silica was taken as 1.45 and that of PMMA as 1.49. The dielectric constant of the silver was taken from Johnson and Christy [5]. The dispersion of the dielectric constant was taken into account during computation. Modeling was done around $600 \mathrm{~nm}$. The WGMs can be identified by the mode number $(\mathrm{m})$ which is half the number of electric field maxima that fit in to the circumference of the resonator and mode order ( $p)$, which is the number of field maxima along the radial direction. Initially we have determined that, for $m=455$ a bare silica sphere has a first order resonance around $600 \mathrm{~nm}$. Therefore, 
for the rest of the modeling we have kept the mode number constant.

This composite cavity exhibits both photonic modes and plasmonic photonic hybrid modes. Fig. 1 show the electric field strength of the symmetric (a) and antisymmetric (b) hybrid modes overlaid with radial electric field profile. The thickness of the polymer layer was $1.2 \mu \mathrm{m}$.
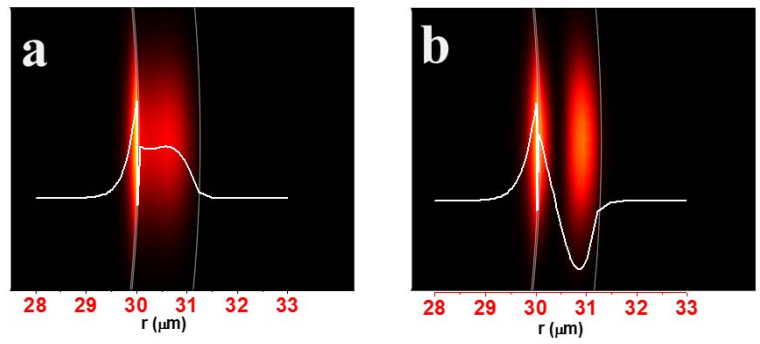

Fig. 1. The symetric (a) and antisymetric hybrid (b) modes of the proposed cavity. The thickness of PMMA was $1.2 \mu \mathrm{m}$. The radial electric field profile is overlaid on the image.

\section{Dependence of resonance on thickness of PMMA}

The resonance frequency of the symmetric and antisymmetric modes as a function of polymer layer thickness is given in Fig 2. When the polymer thickness is $600 \mathrm{~nm}$ the symmetric and antisymmetric mode resonance have a separation of about $34 \mathrm{THz}$. The resonance frequencies come closer as the thickness of the polymer layer increases. The frequency shift of the antisymmetric mode is much higher than that of the symmetric mode. The two modes come closest around a polymer thickness of $1.3 \mu \mathrm{m}$. For polymer layer thicknesses above $1.3 \mu \mathrm{m}$ the resonance frequencies diverge and the symmetric mode redshifts faster than the antisymmetric mode in contrast to the behavior below $1.3 \mu \mathrm{m}$.

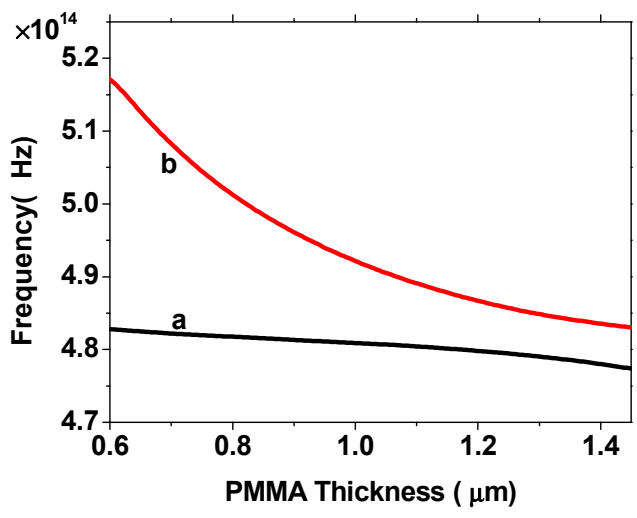

Fig. 2. Variation of the resonance frequency of symetric (a) and antisymetric (b) modes as function of PMMA layer thickness.

\section{Q-factor and mode volume}

The Q-factor and mode volume of the hybrid modes are given in Fig. 3. Both symmetric and antisymmetric modes show $Q$ values more than 1000. The $Q$ factor of the symmetric mode increases with PMMA layer thickness. The antisymmetric mode shows a maximum Q-factor at a layer thickness of $0.9 \mu \mathrm{m}$. The mode volume of the hybrid modes follows the Q-factor curves. This is because when the mode is tightly confined to the metal surface, the absorption from the metal reduces the Q-factor.

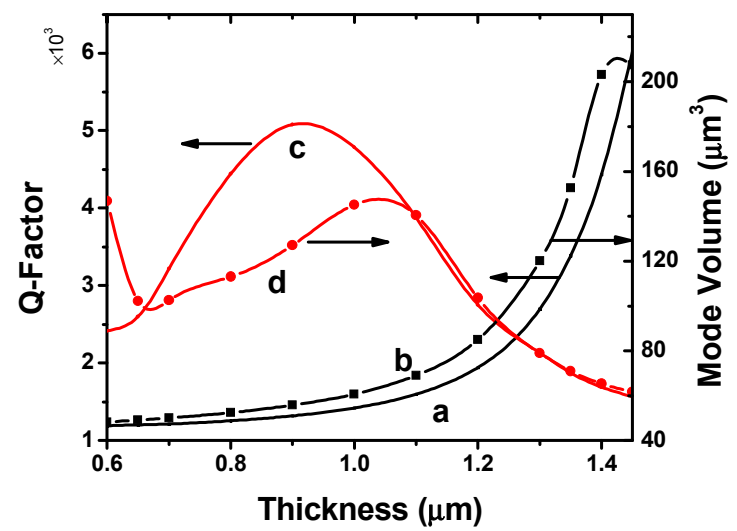

Fig. 3. Q-factor (a) and mode volume (b) of the of symetric mode as a function of PMMA layer thickness. Q-factor (c) and mode volume (d) of antisymetric mode is also shown.

\section{CONCLUSION}

A three-layer hybrid plasmonic WGM resonator is proposed and numerically analyzed. The hybrid plasmonic WGMs show moderately high $Q$ factor and low mode volume. It has been shown that the resonance frequency, mode volume, and Q-factor of the resonance can be tuned by changing the thickness of the outer polymer layer.

\section{ACKNOWLEDGMENT}

We thank P.G Senapathy Centre, IIT Madras for computational resources. We thank EU FP7 Grant under the project "Ultrafast Photonics-Processes and Interactions (UPPI)" for travel funds. PBB thanks DST, New Delhi for financial support.

\section{REFERENCES}

[1] G. M. Akselrod, et al., "Probing the mechanisms of large Purcell enhancement in plasmonic nanoantennas," Nat Photon, vol. 8, pp. 835-840, 2014.

[2] B. Min, et al., "High-Q surface-plasmon-polariton whispering-gallery microcavity," Nature, vol. 457, pp. 455-458, 2009.

[3] A. Rottler, et al., "High-Q Hybrid Plasmon-Photon Modes in a Bottle Resonator Realized with a Silver-Coated Glass Fiber with a Varying Diameter," Physical Review Letters, vol. 111, p. 253901, 2013.

[4] M. Oxborrow, "Traceable 2-D Finite-Element Simulation of the Whispering-Gallery Modes of Axisymmetric Electromagnetic Resonators," IEEE Transactions on Microwave Theory and Techniques, vol. 55, pp. 1209-1218, 2007.

[5] P. B. Johnson and R. W. Christy, "Optical Constants of the Noble Metals," Physical Review B, vol. 6, pp. 4370-4379, 1972. 